\title{
Análise não linear estática de treliça plana utilizando a Mecânica do Dano
}

Nonlinear static analysis of plane truss using the Damage Mechanics

\author{
L. A. F. de Souza \\ Curso de Engenharia Civil, Universidade Tecnológica Federal do Paraná, CEP 86812-460, Apucarana-Pr, Brasil
}

lasouza@utfpr.edu.br

(Recebido em 14 de agosto de 2014; aceito em 29 de dezembro de 2014)

\begin{abstract}
As treliças possuem um vasto campo de aplicação na Engenharia de Estruturas, sendo muito utilizadas na construção de pontes - rodoviárias e ferroviárias, em estruturas de cobertura de edificações, em torres de transmissão de energia, entre diversos outros usos. Em geral são relativamente leves e especialmente indicadas para superar grandes vãos ou suportar carregamentos elevados. Nesse contexto, as treliças tornam-se uma solução econômica e prática. Este artigo tem como objetivo avaliar o comportamento estrutural de treliças planas via Método dos Elementos Finitos considerando a não linearidade física. A relação constitutiva do material é descrita por um modelo baseado na teoria da Mecânica do Dano Contínuo. As análises não lineares são efetuadas por meio do método de Comprimento de Arco com o processo iterativo tipo Newton - Raphson. A partir dos resultados numéricos, a modelagem proposta apresenta potencialidade na análise de treliças planas devido a sua reduzida complexidade e baixo custo computacional.

Palavras-chave: treliça, Mecânica do Dano, elementos finitos
\end{abstract}

The trusses have a wide application in Structural Engineering, being heavily used in the construction of bridges (highway and railway), in roof structures of buildings, towers of energy transmission, among many other uses. Usually they are relatively light weight and especially indicated to overcoming large spans or support high loadings. In this context, the trusses become an economical and practical solution. This paper aims to evaluate the structural behavior of planar trusses by means of Finite Element Method considering the physical nonlinearity. The material constitutive relation is described by a model based on the theory of Continuum Damage Mechanics. The nonlinear analysis is performed considering the Arc Length method with Newton - Raphson iterative process. From the numerical results, the proposed modeling presents a potential capability in the analysis of planar trusses due to its low complexity and low computational cost.

Keywords: truss, Damage Mechanics, finite element

\section{INTRODUÇÃO}

A treliça é uma estrutura constituída por elementos lineares (barras) que podem estar dispostos em diversos planos - estruturas bidimensionais ou tridimensionais. Esta estrutura é muito utilizada na construção de pontes (rodoviárias e ferroviárias), em estruturas de cobertura de edificações, em torres de transmissão de energia, entre diversos outros usos. Usualmente são construídas em madeira ou aço, sendo relativamente leves e indicadas para superar grandes vãos ou suportar carregamentos elevados. Nesse contexto, as treliças tornam-se uma solução econômica e prática [1].

Para efeito de projeto deseja-se conhecer o carregamento último de colapso e sua resposta força aplicada versus deslocamento. Dependendo das características da estrutura, esta resposta pode ser linear ou não linear. No comportamento não linear não há proporcionalidade entre o carregamento aplicado e as deformações e/ou deslocamentos. De maneira geral, pode-se identificar três tipos de comportamento não linear: física, geométrica e de contato [2].

Em treliças planas ou espaciais é comum tratar-se da relação constitutiva para a barra ao invés de relação constitutiva para o material. Neste caso, há uma diferenciação de tratamento ou de comportamento entre barras tracionadas e comprimidas. As barras tracionadas podem atingir 
o escoamento, enquanto as comprimidas podem apresentar instabilidade para tensões inferiores ao escoamento; estas características devem estar inclusas na relação constitutiva da barra.

Os componentes de sistemas estruturais sofrem, ao longo de sua vida útil, alterações que normalmente são dependentes de fatores ambientais e das cargas às quais estão submetidos. Estas alterações, denominadas danos, podem levar ao comprometimento desses componentes. É fundamental que tais danos sejam identificados e avaliados no que concerne as suas localizações e intensidades. Falhas nestas estruturas podem levar a prejuízos materiais e até mesmo à perda de vidas em casos extremos [3].

Fisicamente, o dano pode ser visto como as deformações plásticas ou deformações permanentes originadas pela deterioração das propriedades físicas do material. Conhecer o início, a propagação e as causas do dano é de extrema importância para controlá-lo, estimando assim a vida útil e evitando o colapso de uma estrutura.

Devido ao avanço tecnológico e à utilização de materiais mais resistentes, estruturas mais complexas e esbeltas vêm sendo desenvolvidas, necessitando de métodos computacionais para a sua análise, tendo em vista a dificuldade de se modelar o comportamento real das mesmas com maior precisão. Dessa forma, este artigo apresenta uma proposta de modelagem de estruturas do tipo treliça, por meio do Método dos Elementos Finitos, levando em consideração os efeitos da não linearidade física. O comportamento do material é descrito por um modelo constitutivo baseado na Mecânica do Dano Contínuo proposto por Manzoli (1998) [4]. Ao modelo é incorporado o critério de falha da Máxima Tensão.

As análises não lineares estáticas são efetuadas considerando o método de Comprimento de Arco associado ao processo iterativo tipo Newton - Raphson. Essa técnica se caracteriza por apresentar um controle concomitante de força e deslocamento. As simulações computacionais são realizadas com o programa gratuito Scilab.

Um estudo de um problema de uma treliça plana submetida a uma força incremental crescente, com dano previamente estabelecido em um dos seus elementos, é apresentado. As simulações são conduzidas até a ocorrência da ruptura da estrutura. A partir dos resultados obtidos, a modelagem proposta apresenta potencialidade na análise de estruturas treliçadas devido a sua reduzida complexidade e baixo custo computacional.

\section{MODELO CONSTITUTIVO DO MATERIAL}

Para descrever o comportamento do material, utiliza-se um modelo baseado na Mecânica do Dano Contínuo proposto por Manzoli (1998) [4]. Para o caso de um elemento unidimensional submetido a uma força axial, a tensão efetiva $\bar{\sigma}$ é expressa por:

$$
\bar{\sigma}=\mathrm{E}_{\mathrm{s} 0} \varepsilon
$$

onde $\mathrm{E}_{\mathrm{s} 0}$ é o módulo de elasticidade inicial do material (não danificado), e $\varepsilon$ é a deformação específica. $\mathrm{O}$ limite de dano inicial $\mathrm{r}_{0}$ é uma propriedade do material e pode ser relacionado à tensão limite de proporcionalidade $\mathrm{f}_{0}$ da seguinte forma:

$$
\mathrm{r}_{0}=\mathrm{B}_{1} \frac{\mathrm{f}_{0}}{\sqrt{\mathrm{E}_{\mathrm{s} 0}}}
$$

onde $\mathrm{B}_{1}$ é um parâmetro do modelo. Define-se a norma da tensão efetiva $\tau(\bar{\sigma})$ por:

$$
\tau(\bar{\sigma})=\sqrt{\frac{\bar{\sigma}^{2}}{\mathrm{E}_{\mathrm{s} 0}}}
$$

A partir das relações de Kuhn - Tucker, o limite de dano é dado pelo máximo valor da variável $\tau$ durante o processo de carregamento, tal que: 


$$
\mathrm{r}=\operatorname{máx}\left(\mathrm{r}_{0}, \tau\right)
$$

A partir das Equações (1) e (3), $\tau$ pode ser expresso em termos da deformação $\varepsilon$ por:

$$
\tau(\varepsilon)=\sqrt{\mathrm{E}_{\mathrm{s} 0}} \varepsilon
$$

A tensão normal $\sigma$ no material é obtida da seguinte forma:

$$
\sigma=\left(1-\mathrm{d}_{\mathrm{s}}\right) \mathrm{E}_{\mathrm{s} 0} \varepsilon
$$

onde o dano $\mathrm{d}_{\mathrm{s}}$ é escrito em função do módulo $\mathrm{H}$ e do limite de dano $\mathrm{r}$ por:

$$
\mathrm{d}_{\mathrm{s}}=\frac{\mathrm{r}-\mathrm{r}_{0}}{\mathrm{r}(1+\mathrm{H})}
$$

Variando-se a função que descreve o módulo $\mathrm{H}$, tem-se na Figura 1 em (a) o regime elastodegradável perfeito, em (b) o encruamento linear positivo (endurecimento), em (c) o encruamento linear negativo (abrandamento), e em (d) o abrandamento exponencial.

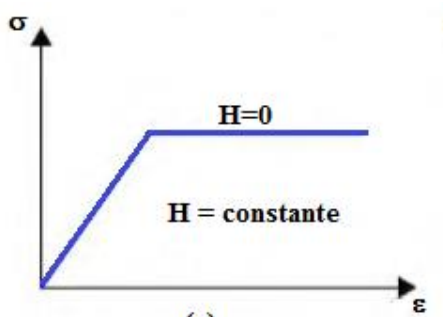

(a)

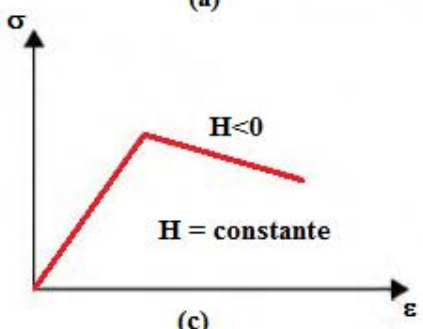

(c)

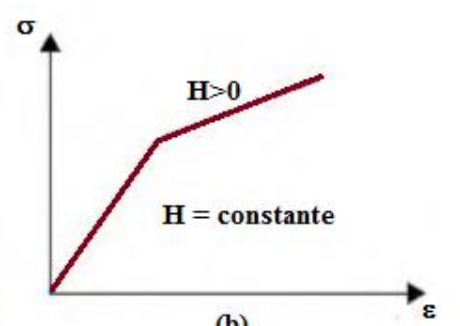

(b)

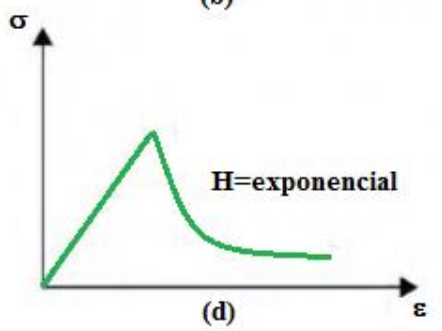

Figura 1: Comportamentos distintos de endurecimento/abrandamento.

Para o caso de um procedimento incremental, o dano $d_{s}$ no passo de carga t pode ser obtido a partir das deformações a cada iteração i pelo algoritmo do modelo constitutivo de dano diferenciado à tração e à compressão, totalmente explícito, apresentado a seguir.

Entrada: $\mathrm{E}_{\mathrm{s} 0}, \mathrm{H}_{\mathrm{c}}, \mathrm{H}_{\mathrm{t}}, \mathrm{r}_{0 \mathrm{c}}, \mathrm{r}_{0 \mathrm{t}}, \varepsilon^{\mathrm{i}}$

1) Calcular $\tau^{\mathrm{i}}=\sqrt{\mathrm{E}_{\mathrm{s} 0}}\left|\varepsilon^{\mathrm{i}}\right|$

2) $\operatorname{Se} \varepsilon^{i}>0 \quad$ (tração)

$$
\text { Se } \tau^{\mathrm{i}>\mathrm{r}_{0 \mathrm{t}}} \mathrm{r}^{\mathrm{i}}=\tau^{\mathrm{i}}
$$

senão

$$
\mathrm{r}^{\mathrm{i}}=\mathrm{r}_{0 \mathrm{t}}
$$

Fim

Fim

Calcular $d_{s}{ }^{i}=\frac{r^{i}-r_{0 t}}{r^{i}\left(1+H_{t}\right)}$

3) $\operatorname{Se} \varepsilon^{\mathrm{i}<0}$

$$
\text { Se } \tau^{\mathrm{i}}>\mathrm{r}_{0 \mathrm{c}}
$$

(compressão) 
Fim

$$
\text { senão } \begin{aligned}
& \mathrm{r}^{\mathrm{i}}=\tau^{\mathrm{i}} \\
& \mathrm{r}^{\mathrm{i}}=\mathrm{r}_{0 \mathrm{c}}
\end{aligned}
$$

Fim

Calcular $d_{s}^{i}=\frac{r^{i}-r_{0 c}}{r^{i}\left(1+H_{c}\right)}$

4) $\quad$ Se $\varepsilon^{\mathrm{i}}=0, \mathrm{~d}_{\mathrm{s}}^{\mathrm{i}}=0$, Fim

5) $\quad$ Se $d_{s}{ }^{i}>1, d_{s}{ }^{i}=1$, Fim

6) $\quad \operatorname{Se~d}_{\mathrm{s}}{ }^{\mathrm{i}}<0, \mathrm{~d}_{\mathrm{s}}{ }^{\mathrm{i}}=0$, Fim

Saída: $\mathrm{d}_{\mathrm{s}}^{\mathrm{i}}$

No algoritmo, os limites de dano inicial $\mathrm{r}_{0 \mathrm{c}}$ e $\mathrm{r}_{0 \mathrm{t}}$ são referentes à compressão e à tração, respectivamente, e os módulos $\mathrm{H}_{\mathrm{c}}$ e $\mathrm{H}_{\mathrm{t}}$ referem-se à compressão e à tração, respectivamente.

\section{CRITÉRIO DE FALHA}

Ao modelo é incorporado o critério de falha da Máxima Tensão. A falha da barra ocorre quando a seguinte inequação for satisfeita:

$$
\mathrm{B}_{2}\left(\frac{\sigma_{1}}{\sigma_{\text {últ }}}\right)^{2} \geq 1
$$

onde $\sigma_{1}$ é a tensão principal na direção $1, \sigma_{\text {últ }}$ é a tensão última à tração ou à compressão na direção 1 , e $\mathrm{B}_{2}$ é uma constante.

\section{MÉTODO DE COMPRIMENTO DE ARCO COM O PROCESSO ITERATIVO TIPO NEWTON - RAPHSON}

Para problemas de ponto limite, ao aplicar o método de Newton - Raphson com controle de força, a matriz de rigidez tende a singularizar nas proximidades desse ponto em sua trajetória ascendente. Uma alternativa para detectar e ultrapassar o ponto limite é a utilização de métodos de solução associados ao método de Newton - Raphson, como por exemplo, o método de Comprimento de Arco. O método de Comprimento de Arco caracteriza-se por apresentar um controle concomitante de força e deslocamento. Há duas incógnitas: o incremento do fator de força $\Delta \varphi$ e o vetor de incremento de deslocamento $\Delta \mathbf{u}$. Em cada passo de solução, as trajetórias de iteração são perpendiculares aos arcos, que por sua vez podem ser aproximados por tangentes à trajetória de equilíbrio [5]. Considerando o método Comprimento de Arco com o processo de iteração tipo Newton - Raphson, as equações de equilíbrio para i - ésima iteração podem ser escritas como:

$$
\mathbf{K}_{\mathbf{T}} \Delta \mathbf{u}^{\mathbf{i}}=\Delta \varphi^{\mathrm{i}} \mathbf{R}_{\mathbf{0}}+\Delta \mathbf{Q}^{\mathbf{i}-\mathbf{1}}
$$

onde $\Delta \varphi^{\mathrm{i}}$ é o incremento do fator de força da iteração i, $\Delta \mathbf{u}^{\mathbf{i}}$ é o vetor incremento de deslocamento, $\mathbf{R}_{\mathbf{0}}$ é o vetor de forças de referência, $\mathbf{K}_{\mathbf{T}}$ é a matriz de rigidez tangente atualizada a cada iteração, e $\Delta \mathbf{Q}^{\mathbf{i}-\mathbf{1}}$ é o vetor de forças não equilibradas dado por:

$$
\Delta \mathbf{Q}^{\mathrm{i}-1}=\mathbf{R}_{\mathrm{ext}}^{\mathrm{i}-1}-\mathbf{F}_{\mathrm{int}}^{\mathrm{i}}
$$

onde $\mathbf{R}_{\text {ext }}{ }^{\mathbf{i}-\mathbf{1}}$ é o vetor de forças externas, e $\mathbf{F}_{\mathbf{i n t}}{ }^{\mathbf{i}}$ é o vetor de forças internas. O vetor $\mathbf{R}_{\mathbf{e x t}}{ }^{\mathbf{i}-\mathbf{1}}$ deve ser escrito em função do fator de força $\varphi^{\mathbf{i}-1}$, atualizado ao final da iteração anterior e do vetor de forças de referência $\mathbf{R}_{\mathbf{0}}$, constante, por meio da seguinte relação: 


$$
\mathbf{R}_{\text {ext }}{ }^{\mathbf{i}-1}=\varphi^{\mathrm{i}-1} \mathbf{R}_{\mathbf{0}}
$$

Para um sistema de ordem $\mathrm{n}+1$, equivalendo $\mathrm{n}$ ao número de graus de liberdade da estrutura, tem-se:

$$
\left[\begin{array}{cc}
\mathbf{K}_{\mathbf{T}} & -\mathbf{R}_{\mathbf{0}} \\
\Delta \mathbf{u}^{\mathbf{1}} & \Delta \varphi^{1}
\end{array}\right]\left\{\begin{array}{c}
\Delta \mathbf{u}^{\mathrm{i}} \\
\Delta \varphi^{\mathrm{i}}
\end{array}\right\}=\left\{\begin{array}{c}
\Delta \mathbf{Q}^{\mathbf{i}-\mathbf{1}} \\
0
\end{array}\right\}
$$

onde $\Delta \mathbf{u}^{1}$ é o primeiro vetor de incrementos de deslocamento do passo de solução, e $\Delta \varphi^{1}$ é o primeiro incremento do fator de força no referido passo. Nota-se que a resolução do sistema dado em (12) gera um sistema de equações com solução não trivial mesmo que a matriz $\mathbf{K}_{\mathbf{T}}$ seja singular, o que representa grande vantagem para a solução de problemas com ponto limite.

Entretanto, há o problema da matriz de rigidez não ser simétrica. A fim de contornar esse problema, Wessels (1977) [6] propôs uma alternativa que consiste em dividir o vetor de incremento de deslocamentos $\Delta \mathbf{u}^{\mathbf{i}}$ em duas parcelas: $\Delta \mathbf{u}^{\mathbf{i}}{ }_{\mathbf{Q}}$ e $\Delta \mathbf{u}^{\mathbf{i}}{ }_{\mathbf{R}}$. Essas parcelas são obtidas pela solução dos sistemas:

$$
\begin{aligned}
& \mathbf{K}_{T} \Delta \mathbf{u}^{\mathbf{i}}=\Delta \mathbf{Q}^{\mathbf{i}-\mathbf{1}} \\
& \mathbf{K}_{\mathrm{T}} \Delta \mathbf{u}^{\mathbf{i}}{ }_{\mathbf{R}}=\mathbf{R}_{\mathbf{0}}
\end{aligned}
$$

O incremento do fator de força é calculado por:

$$
\Delta \varphi^{\mathrm{i}}=-\frac{\Delta \mathbf{u}^{\mathbf{1}}: \Delta \mathbf{u}_{\mathbf{Q}}^{\mathbf{i}}}{\Delta \mathbf{u}^{\mathbf{1}}: \Delta \mathbf{u}^{\mathrm{i}}{ }_{\mathbf{R}}+\Delta \varphi^{1}}
$$

Com o objetivo de limitar os processos iterativos, dois critérios de convergência são estabelecidos: um para os deslocamentos e outro para as forças.

O critério de convergência para os deslocamentos deve obedecer a seguinte desigualdade:

$$
\frac{\left\|\Delta \mathbf{u}^{\mathbf{i}}\right\|}{\left\|\mathbf{u}^{\mathbf{i}}\right\|} \leq \mathrm{u}_{\mathrm{tol}}
$$

onde o numerador é a norma euclidiana do vetor de incremento de deslocamento $\Delta \mathbf{u}^{\mathbf{i}}$ correspondente à iteração i, e o denominador é a norma euclidiana do vetor de deslocamento total $\mathbf{u}^{\mathrm{i}}=\mathbf{u}^{\mathrm{i}-1}+\Delta \mathbf{u}^{\mathrm{i}}$ da $\mathrm{i}$ - ésima iteração.

$\mathrm{O}$ critério de convergência para forças deve obedecer à relação:

$$
\frac{\left\|\Delta \mathbf{Q}^{\mathbf{i}}\right\|}{\left\|\varphi^{\mathrm{i}} \mathbf{R}_{\mathbf{0}}\right\|} \leq Q_{\mathrm{tol}}
$$

onde o numerador é a norma euclidiana do incremento de força não equilibrada $\Delta \mathbf{Q}^{\mathbf{i}}$ correspondente à iteração $\mathrm{i}$, e o denominador é a norma euclidiana do incremento de força do passo de solução.

\section{MATRIZ DE RIGIDEZ E VETOR DE FORÇA INTERNA DO ELEMENTO FINITO DE TRELIÇA PLANA}

A matriz de rigidez do elemento finito de treliça $\mathbf{K}_{\mathrm{e}}$ é modificada para incorporar a variável de dano $\mathrm{d}_{\mathrm{s}}$, ao longo da análise numérica, de acordo com a seguinte equação no sistema de coordenadas globais: 


$$
\mathbf{K}_{\mathbf{e}}=\frac{\left(1-\mathrm{d}_{\mathrm{S}}\right) \mathrm{E}_{\mathrm{S} 0} \mathrm{~A}_{0}}{\mathrm{~L}_{0}}\left[\begin{array}{rrrr}
\mathrm{C}^{2} & \mathrm{CS} & -\mathrm{C}^{2} & -\mathrm{CS} \\
\mathrm{CS} & \mathrm{S}^{2} & -\mathrm{CS} & -\mathrm{S}^{2} \\
-\mathrm{C}^{2} & -\mathrm{CS} & \mathrm{C}^{2} & \mathrm{CS} \\
-\mathrm{CS} & -\mathrm{S}^{2} & \mathrm{CS} & \mathrm{S}^{2}
\end{array}\right]
$$

onde $\mathrm{A}_{0}$ é a área da seção transversal indeformada, $\mathrm{L}_{0}$ é o comprimento indeformado, $\mathrm{E}_{\mathrm{s} 0}$ é o módulo de elasticidade não danificado (material íntegro), e $d_{s}$ é o dano. Na Equação (18) as variáveis $\mathrm{S}$ e $\mathrm{C}$ denotam $\operatorname{sen}(\alpha)$ e $\cos (\alpha)$, respectivamente.

$\mathrm{Na}$ determinação das tensões em cada barra da treliça é necessário determinar as forças internas. Estas forças em um elemento de nós i e j, representado na Figura 2, são dadas por:

$$
\begin{aligned}
& f_{x i}=\frac{\left(1-d_{s}\right) E_{s 0} A}{L}\left(u_{x i}-u_{x j}\right) \\
& f_{x j}=\frac{\left(1-d_{s}\right) E_{s 0} A}{L}\left(u_{x j}-u_{x i}\right)
\end{aligned}
$$

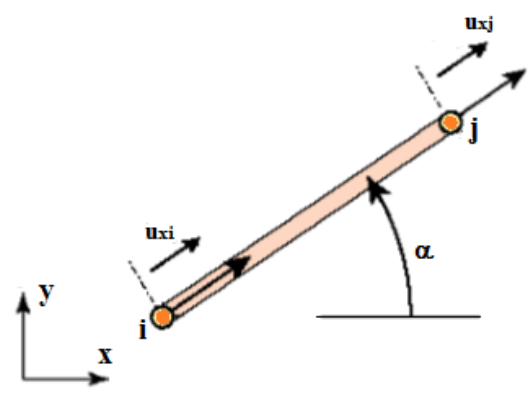

Figura 2: Representação de um elemento finito de treliça plana.

No entanto, para a determinação dessas forças é preciso que se tenha o valor dos deslocamentos no sistema de coordenadas locais. Os deslocamentos locais u são determinados por:

$$
\mathbf{u}=\mathbf{T}^{-1} \mathbf{U}
$$

onde $\mathbf{U}$ é o vetor de deslocamentos nodais no sistema de coordenadas globais, e $\mathbf{T}$ é a matriz de rotação. $\mathrm{O}$ vetor de força interna elementar $\mathbf{F}_{\text {inte }}$ no sistema de coordenadas locais é dado por:

$$
F_{\text {inte }}=\left[\begin{array}{c}
f_{x i} \\
0 \\
f_{x j} \\
0
\end{array}\right]
$$

\section{RESULTADOS E DISCUSSÃO}

\subsection{SIMULAÇÃO 1}

Este exemplo numérico tem por objetivo verificar o modelo de treliça plana implementado neste trabalho. A treliça indicada na Figura 3 foi estudada por Leite (2000) [7] e consiste de três barras DA, DB e DC. Todas têm o módulo de elasticidade $\mathrm{E}_{\mathrm{s} 0}=20500 \mathrm{kN} / \mathrm{cm}^{2}$, tensão limite de escoamento $\mathrm{f}_{0}=34,50 \mathrm{kN} / \mathrm{cm}^{2}$, e área da seção transversal $\mathrm{A}_{0}=12,51 \mathrm{~cm}^{2}$. Uma força concentrada $\mathrm{P}$ é aplicada no nó $\mathrm{D}$ e os parâmetros geométricos comprimento $\mathrm{L}$ e ângulo $\beta$ apresentam os valores $200 \mathrm{~cm}$ e $45^{\circ}$, respectivamente. Na simulação considera-se os módulos de endurecimento $\mathrm{H}_{\mathrm{c}}$ e $\mathrm{H}_{\mathrm{t}}$ constantes e iguais a $10^{-6}$ (regime elastodegradável perfeito) e $\mathrm{B}_{1}=1$. $\mathrm{O}$ incremento de força utilizado é igual a $21,0 \mathrm{kN}$ e os erros máximos admitidos no final de cada iteração são de $\mathrm{u}_{\mathrm{tol}}=10^{-2} \mathrm{e} \mathrm{Q}_{\mathrm{tol}}=10^{-2}$. O peso próprio é desprezado nesta simulação. 


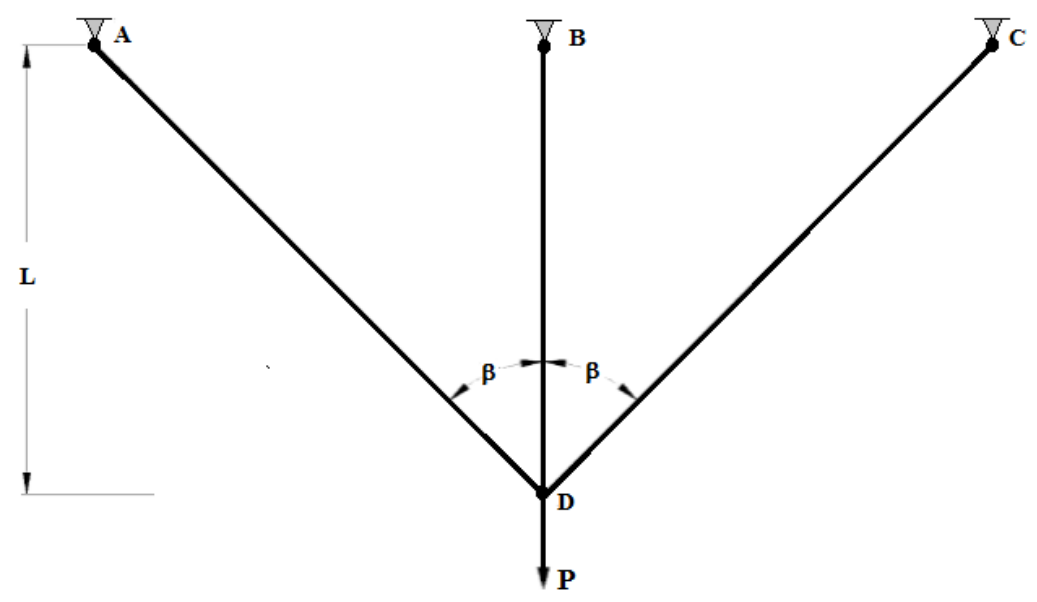

Figura 3: Esquema estrutural da treliça plana com três barras.

Na Figura 4 são apresentadas as curvas deslocamento vertical no nó D versus força, comparando os resultados obtidos pela modelagem proposta com os resultados teóricos e numéricos apresentados por Leite (2000) [7]. Vê-se nesta figura que os resultados obtidos com a modelagem proposta apresentou boa concordância com os demais. Todas as barras trabalham em regime elástico linear até cerca de $70 \%$ da força aplicada $(\mathrm{P} \cong 735 \mathrm{kN})$. A partir desse incremento de força somente a barra BD escoa $\left(\mathrm{d}_{\mathrm{s}}>0\right)$ diminuindo a rigidez do sistema, conforme indica a mudança de inclinação da curva numérica. Neste trabalho, considera-se que a treliça falha quando ocorre o escoamento das três barras. Assim, a convergência na simulação acontece para $\mathrm{P}=1029,0031 \mathrm{kN}$, aproximadamente.

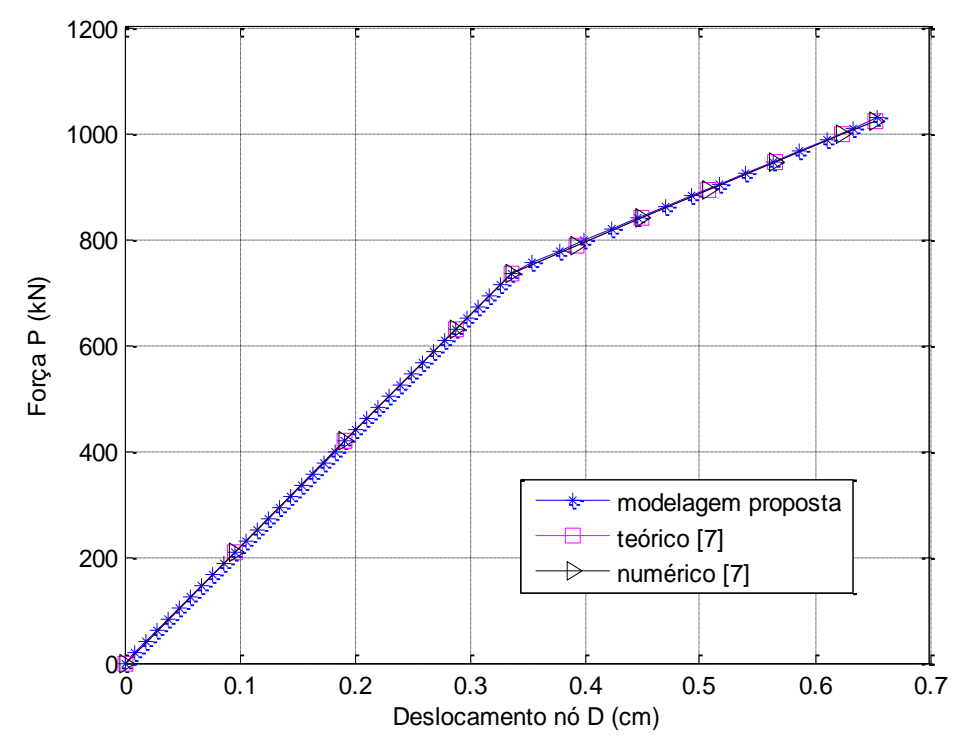

Figura 4: Curvas deslocamento vertical no nó D versus força $P$.

\subsection{SIMULAÇÃO 2}

A estrutura da treliça plana hiperestática mostrada na Figura 5 é composta por elementos que possuem mesmo módulo de elasticidade $\left(\mathrm{E}_{\mathrm{s} 0}=20500 \mathrm{kN} / \mathrm{cm}^{2}\right)$, sendo que os banzos superior e inferior são constituídos por perfis laminados em cantoneira de abas iguais 4 " x 4 " x $1 / 4$ " $\left(\mathrm{A}_{0} \cong\right.$ $\left.12,51 \mathrm{~cm}^{2}\right)$ e as diagonais e montantes por perfis laminados em cantoneira de abas iguais $11 / 2$ " x $11 / 2$ " x $3 / 16$ " $\left(\mathrm{A}_{0} \cong 3,42 \mathrm{~cm}^{2}\right)$. Os elementos apresentam tensão de escoamento $\mathrm{f}_{0}=180 \mathrm{MPa}$ e tensão última (ruptura) $\sigma_{\text {últ }}=330 \mathrm{MPa}$, tanto à tração quanto à compressão. O peso próprio é desprezado nas simulações. 


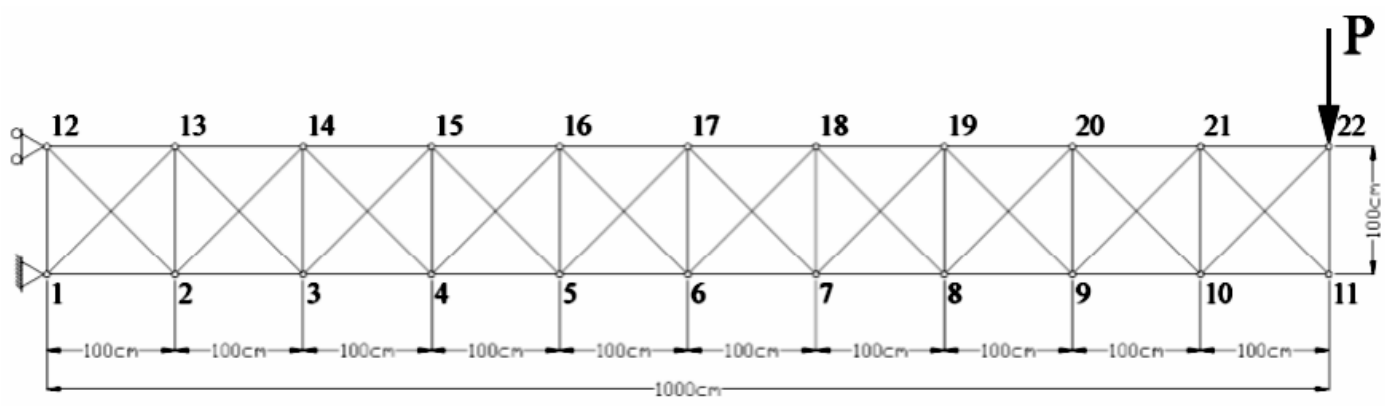

Figura 5: Treliça plana hiperestática em balanço.

$\mathrm{Na}$ análise não linear estática da estrutura, adotou-se o modelo de dano isotrópico com os valores do módulos $\mathrm{H}_{\mathrm{c}}$ e $\mathrm{H}_{\mathrm{t}}$ iguais a 0,1 (encruamento linear positivo) e $\mathrm{B}_{1}=1$. Para o critério de falha da Máxima Tensão, considerou-se $\mathrm{B}_{2}=1$. O incremento de força utilizado é igual a $0,88 \mathrm{kN}$ e os erros máximos admitidos no final de cada incremento são $\mathrm{u}_{\mathrm{tol}}=10^{-3} \mathrm{e}_{\mathrm{tol}}=10^{-3}$.

Na Figura 6 são apresentadas as curvas deslocamento vertical no nó 22 versus força, considerando o elemento 1-2, localizado no banzo inferior da treliça, íntegro $\left(d_{s}=0\right)$ e danificado previamente $\left(d_{s}=0,25\right.$ e 0,5$)$.

Fazendo-se a análise dessa figura, vê-se que com o dano previamente estabelecido na barra 12 a resposta da estrutura é alterada quanto à força de ruptura e ao deslocamento vertical correspondente. As simulações foram conduzidas até o colapso global da estrutura (no momento em que a barra 1-2 falha, isto é, quando a relação $\left(\frac{\sigma_{1}}{\sigma_{u ́ l t}}\right)^{2} \geq 1$ é satisfeita na verificação do critério de resistência para o elemento).

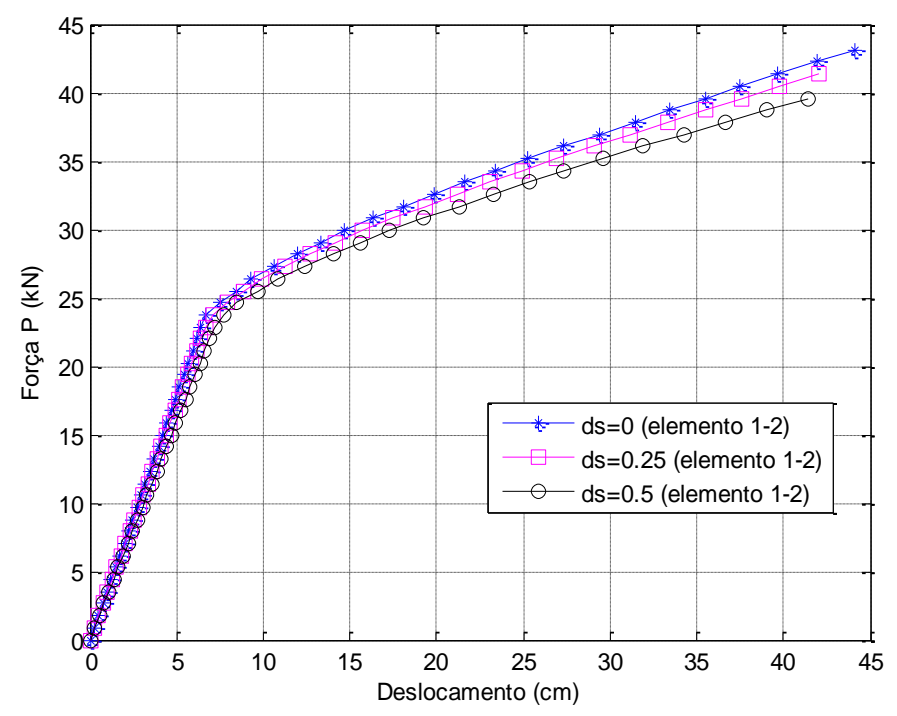

Figura 6: Curvas deslocamento vertical no nó 22 versus força.

Na Figura 7 é apresentada a curva deslocamento vertical no nó 22 versus força até a falha global da estrutura na simulação (instabilidade na convergência), para o caso em que a barra 1-2 não está previamente danificada $\left(\mathrm{d}_{\mathrm{s}}=0\right)$. 


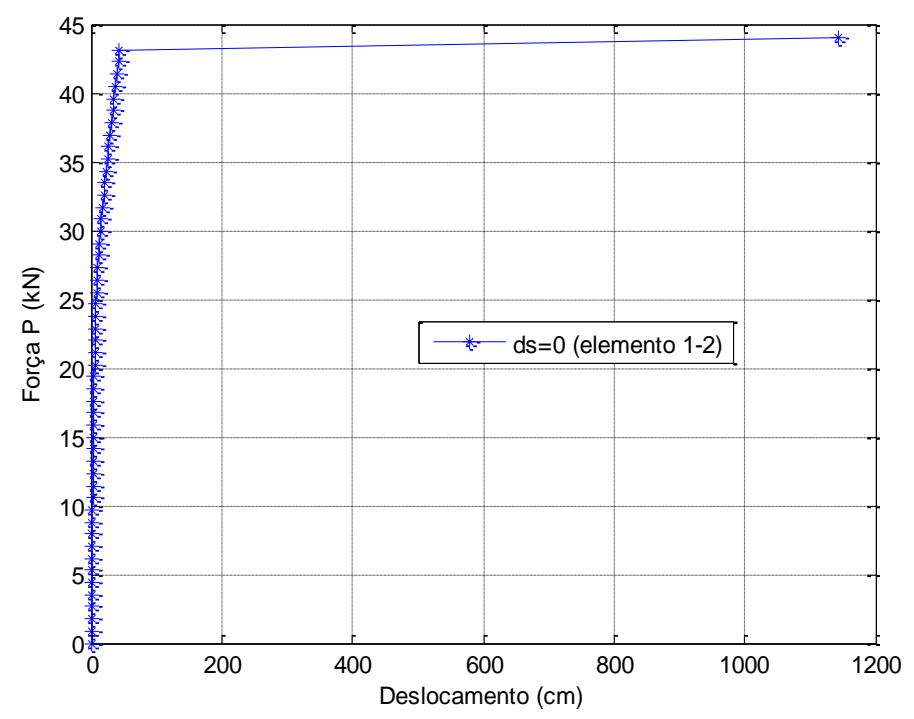

Figura 7: Curva deslocamento vertical no nó 22 versus força até o colapso da estrutura.

\section{CONCLUSÃO}

A modelagem proposta, utilizando o Método dos Elementos Finitos e o modelo constitutivo implementado, apresenta potencialidade na simulação numérica do comportamento estrutural de treliças planas devido a sua reduzida complexidade e baixo custo computacional.

Foram utilizadas treliças planas submetidas a carregamentos estáticos. Verificou-se na segunda simulação que análises numéricas com elementos previamente danificados possibilitam ao projetista avaliar as possíveis influências desses elementos sobre a resposta mecânica da estrutura de treliça.

Observa-se que a análise com modelos constitutivos baseados na Mecânica do Dano Contínuo constitui uma válida alternativa para o estudo do comportamento estrutural. No entanto, a aplicação de tais modelos em situações práticas fica prejudicada pela identificação experimental dos parâmetros contidos nas leis de evolução das variáveis de dano.

Uma barreira considerável no uso corrente de modelos não lineares está relacionada aos problemas numéricos e instabilidades na convergência que podem surgir. Isso ocorre por conta da calibração dos parâmetros necessários nos modelos não lineares, sendo oneroso o processo, visto que diversas análises preliminares devem ser feitas antes de realizar o estudo final da estrutura.

Para desenvolvimentos futuros, sugere-se a implementação de algoritmos que permitam efetuar ciclos de carregamento e descarregamento, a consideração da não linearidade geométrica e da flambagem nas análises e, também, adequar o código implementado para estudos em análise dinâmica.

\section{AGRADECIMENTOS}

Agradeço a UTFPR pelo apoio financeiro para a realização desta pesquisa.

\section{REFERÊNCIAS BIBLIOGRÁFICAS}

1. Kripka M, Pravia ZMC, Dias MM, Medeiros GF. Minimização do peso de treliças de alumínio pela otimização simultânea da seção transversal dos elementos e da geometria: análise numérica e validação experimental. Revista Eletrônica de Engenharia Civil - REEC. 2013; 7(2): 19-26. 
2. Martins TV, Greco M, Lemonge ACC. Otimização paramétrica de estruturas treliçadas com objetivo baseado no comportamento pós crítico via algoritmos genéticos. In: XI Simpósio de Mecânica Computacional. Anais do XI Simpósio de Mecânica Computacional; 2014; Juiz de Fora.

3. Santos GF, Silva JGS, Soeiro FJCP, Neto AJSN. Identificação de dano e análise de sensibilidade em treliças de aço. In: Mecánica Computacional, Vol. XXIX. Anais de Mecánica Computacional, Vol. XXIX; 2010; Buenos Aires.

4. Manzoli OL. Um modelo analítico y numérico para la simulatión de discontinuidades fuertes em la mecânica de sólidos [doutorado]. Barcelona: Escola Técnica Superior D’Enginyers de Camins, Canals i Ports, Universitat Politécnica de Catalunya; 1998.

5. Ramm E. Strategies for tracing the nonlinear response near limit points. In: Euro-US-Workshop on Nonlinear Finite Element Analysis in Structural Mechanics. Proceedings of Euro-US-Workshop on Nonlinear Finite Element Analysis in Structural Mechanics, Ruhr Universitat Bochum; 1981; Berlin: Springer; p. 68-89.

6. Wessels M. Das Statische und Dynamishe Durchschlagsproblem der Imperfektem Flachen Kugelchale bei Elasticher Rotatiossymmetrischer Verformumg [dissertation]. Hannover: T. U. Hannover, Mitteil, n. 23 des Imstituts für Statik; 1977.

7. Leite FN. Uma Formulação Teórica Consistente para Análise Não Linear de Estruturas Treliçadas Espaciais. Dissertação [mestrado]. Belo Horizonte: Universidade Federal de Minas Gerais, Escola de Engenharia Departamento de Engenharia de Estruturas; 2000. 